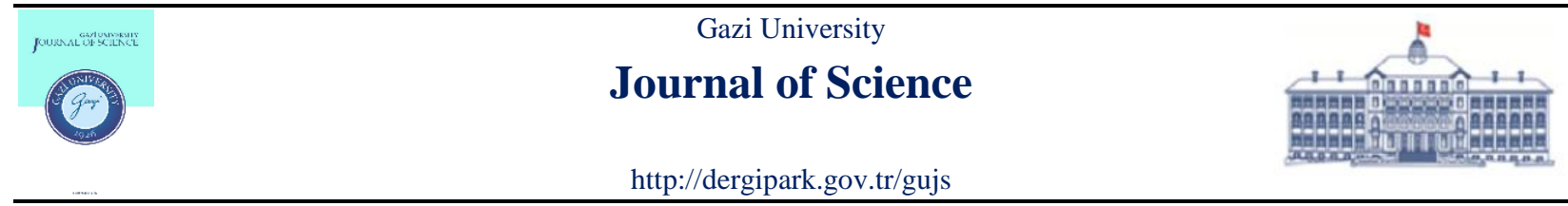

\title{
A Study on Dual-Generalized Complex and Hyperbolic-Generalized Complex Numbers
}

\author{
Nurten GURSES ${ }^{*(D)}$, Gulsum Yeliz SENTURK ${ }^{2}$, Salim YUCE $^{1}$ (D) \\ ${ }^{1} Y i l d i z$ Technical University, Faculty of Arts and Sciences, Department of Mathematics, 34220, Esenler, Istanbul, Turkey \\ ${ }^{2}$ Istanbul Gelisim University, Faculty of Engineering and Architecture, Department of Computer Engineering, 34310, Avclar, Istanbul, Turkey

\section{Highlights} \\ - This paper focuses on the theories of dual-generalized and hyperbolic-generalized complex numbers. \\ - The algebraic structures of dual-generalized and hyperbolic-generalized complex numbers are given. \\ - Dual-generalized complex and hyperbolic-generalized complex valued functions are defined. \\ - The matrix representations of dual-generalized and hyperbolic-generalized complex numbers are stated. \\ - An efficient classification includes complex-generalized complex numbers is examined.
}

\section{Article Info}

Received:02/12/2019

Accepted: 28/08/2020

\section{Keywords}

Generalized complex

numbers

Dual-generalized

Complex nnumber

Hyperbolic-generalized

Complex number

\begin{abstract}
This work is intended to introduce the theories of dual-generalized complex and hyperbolicgeneralized complex numbers. The algebraic properties of these numbers are taken into consideration. Besides, dual-generalized complex and hyperbolic-generalized complex valued functions are defined and different matrix representations of these numbers are examined. Moreover, a remarkable classification are given for special cases and the set of complexgeneralized complex numbers are mentioned.
\end{abstract}

\section{INTRODUCTION}

The set of complex numbers is denoted by $\mathbb{C}$ and defined as

$$
\mathbb{C}=\left\{z=\left(x_{1}, x_{2}\right)=x_{1}+x_{2} i \mid x_{1}, x_{2} \in \mathbb{R}, i^{2}=-1\right\} .
$$

As it is well known that the set of complex numbers is a field, an associative and commutative algebra with unity. The complex numbers are applied to many aspects of real life, especially in applied mathematics, physics, quantum mechanics and electronics. The detailed information related to complex numbers can be found in [1].

Besides, different definitions of the complex unit " $i$ " conduce to new special number systems. Hence complex numbers are the building blocks of the other two dimensional number systems, such as hyperbolic numbers (double, split complex, perplex) [2,3], dual numbers [4,5] and multicomponent number systems [6].

As a generalization of complex numbers, the set of generalized complex numbers is denoted by $\mathbb{C}_{\mathfrak{p}}$ and defined as: 


$$
\mathbb{C}_{\mathfrak{p}}=\left\{z=\left(x_{1}, x_{2}\right)=x_{1}+x_{2} J \mid x_{1}, x_{2} \in \mathbb{R}, J^{2}=\mathfrak{p},-\infty<\mathfrak{p}<\infty\right\} \text {. }
$$

The generalized complex numbers analogous with the set of complex numbers $\mathbb{C}$ for $\mathfrak{p}=-1$, hyperbolic numbers $\mathbb{H}$ for $\mathfrak{p}=1$, dual numbers $\mathbb{D}$ for $\mathfrak{p}=0$. Also, $\mathbb{C}_{\mathfrak{p}}$ is a vector space over real numbers $\mathbb{R}$ and $\{1, J\}$ is basis $\mathbb{C}_{\mathfrak{p}}$. The further information about generalized complex numbers can be found in [7].

The idea of investigating the number systems by writing the coefficients as elements of the sets $\mathbb{C}, \mathbb{H}$ and $\mathbb{D}$ is a remarkable area for the researchers. Therefore, hyperbolic numbers with complex coefficients (complex-hyperbolic numbers or hyperbolic-complex numbers) are introduced by [8] and examined in [9, 10]. $n$-dimensional hyperbolic-complex numbers are examined in [11]. Moreover, bicomplex numbers are investigated in [12-14] . Dual numbers with complex coefficients (complex-dual numbers or dual-complex numbers) are presented in [15, 16]. In [17], the concept of dual-complex numbers and their holomorphic functions are investigated. Moreover, the algebraic properties, functions and matrix representations of dualhyperbolic numbers and complex-hyperbolic numbers are presented in [18]. Also, as an extension of dual numbers, hyperdual numbers are studied in [19-24]. Bihyperbolic numbers are examined in [25-28]. There exist quite studies in the literature related to types of numbers which consist of different combination of the coefficients, $[29,30]$.

The main point of this paper is to develop the theory of dual-generalized complex and hyperbolicgeneralized complex numbers by using generalized complex number theory. So, the paper is organized as 3 main sections and conclusions section. Section 2 is devoted to the construction of the concept of dualgeneralized complex numbers. In this section, we firstly introduce algebraic structures of dual-generalized complex numbers such as the conjugations and the modules. Then, dual-generalized complex valued functions are concerned. Besides, the real and generalized matrix representations of dual-generalized complex numbers and the real and dual matrix representations of generalized complex-dual numbers are stated. In Section 3, the concept of hyperbolic-generalized complex numbers are given. The algebraic structures, hyperbolic-generalized complex valued functions, and matrix representations of these numbers are concerned. In conclusions, a notable classification that includes complex-generalized complex numbers are addressed by considering special cases.

\section{DUAL-GENERALIZED COMPLEX NUMBERS}

In this original section, we construct the theory of dual-generalized complex numbers.

Definition 1. A dual-generalized complex number $w$ is an ordered pair of generalized complex numbers $\left(z_{1}, z_{2}\right)$ and denoted in the form

$w=\left(z_{1}, z_{2}\right)=z_{1}+z_{2} \varepsilon$,

where $z_{1}=x_{1}+x_{2} J \in \mathbb{C}_{\mathfrak{p}}, z_{2}=y_{1}+y_{2} J \in \mathbb{C}_{\mathfrak{p}}, J^{2}=\mathfrak{p},-\infty<\mathfrak{p}<\infty$ and $\varepsilon^{2}=0, \varepsilon \neq 0$. This special number is a dual number with generalized complex coefficients. The set of these numbers is denoted by $\mathbb{D} \mathbb{C}_{\mathfrak{p}}$ and defined as

$\mathbb{D C}_{\mathfrak{p}}=\left\{w=z_{1}+z_{2} \varepsilon \mid z_{1}, z_{2} \in \mathbb{C}_{\mathfrak{p}}, \varepsilon^{2}=0, \varepsilon \neq 0\right\}$

If $x_{1} \neq 0, x_{2} \neq 0$ and $y_{1}=y_{2}=0$, then $x_{1}+x_{2} J$ is called proper generalized-complex number. If all values are non-zero, $w$ is called a proper dual-generalized complex number.

Definition 2. Let $w_{1}, w_{2} \in \mathbb{D} C_{\mathfrak{p}}$ and $\lambda \in \mathbb{R}$. Then, the addition, substraction, multiplication, scalar multiplication and equality of dual-generalized complex numbers are defined respectively as in the following Table: 
Table 1. Operations for dual-generalized complex numbers

\begin{tabular}{|c|c|c|}
\hline Addition & $\begin{aligned}: \mathbb{D} \mathbb{C}_{\mathfrak{p}} \times \mathbb{D} \mathbb{C}_{\mathfrak{p}} & \rightarrow \mathbb{D C}_{\mathfrak{p}} \\
\left(w_{1}, w_{2}\right) & \rightarrow w_{1} \oplus w_{2}\end{aligned}$ & $\begin{aligned} w_{1} \oplus w_{2} & =\left(z_{11}+z_{12} \varepsilon\right) \oplus\left(z_{21}+z_{22} \varepsilon\right) \\
& =\left(z_{11}+z_{21}\right)+\left(z_{12}+z_{22}\right) \varepsilon\end{aligned}$ \\
\hline Substraction & $\begin{aligned} \ominus: \mathbb{D} \mathbb{C}_{\mathfrak{p}} \times \mathbb{D} \mathbb{C}_{\mathfrak{p}} & \rightarrow \mathbb{D} \mathbb{C}_{\mathfrak{p}} \\
\left(w_{1}, w_{2}\right) & \rightarrow w_{1} \ominus w_{2}\end{aligned}$ & $\begin{aligned} w_{1} \ominus w_{2} & =\left(z_{11}+z_{12} \varepsilon\right) \ominus\left(z_{21}+z_{22} \varepsilon\right) \\
& =\left(z_{11}-z_{21}\right)+\left(z_{12}-z_{22}\right) \varepsilon\end{aligned}$ \\
\hline Multiplication & $\begin{aligned} \Delta: \mathbb{D} \mathbb{C}_{\mathfrak{p}} \times \mathbb{D C}_{\mathfrak{p}} & \rightarrow \mathbb{D C}_{\mathfrak{p}} \\
\left(w_{1}, w_{2}\right) & \rightarrow w_{1} \Delta w_{2}\end{aligned}$ & $\begin{aligned} w_{1} \Delta w_{2} & =\left(z_{11}+z_{12} \varepsilon\right) \Delta\left(z_{21}+z_{22} \varepsilon\right) \\
& =\left(z_{11} z_{21}\right)+\left(z_{11} z_{22}+z_{12} z_{21}\right) \varepsilon\end{aligned}$ \\
\hline Scalar Multiplication & $\begin{aligned} \odot: \mathbb{R} \times \mathbb{D} \mathbb{C}_{\mathfrak{p}} & \rightarrow \mathbb{D} \mathbb{C}_{\mathfrak{p}} \\
\left(\lambda, w_{1}\right) & \rightarrow \lambda \odot w_{1}\end{aligned}$ & $\begin{aligned} \lambda \odot w_{1} & =\lambda \odot\left(z_{11}+z_{12} \varepsilon\right) \\
& =\left(\lambda z_{11}\right)+\left(\lambda z_{12}\right) \varepsilon\end{aligned}$ \\
\hline Equality & \multicolumn{2}{|c|}{$w_{1}=w_{2} \Leftrightarrow z_{11}+z_{12} \varepsilon=z_{21}+z_{22} \varepsilon \Leftrightarrow z_{11}=z_{21} \wedge z_{12}=z_{22}$} \\
\hline
\end{tabular}

Proposition 1. The algebraic properties of $\mathbb{D C}_{\mathfrak{p}}$ are given as follows:

i) $\quad\left(\mathbb{D C}_{\mathfrak{p}}, \oplus, \Delta\right)$ is a commutative ring with unity under addition and multiplication as defined in Definition 2. The additive and multiplicative identities of this ring are $(0,0)=0+0 \varepsilon$ and $(1,0)=1+0 \varepsilon$, respectively. But, $\mathbb{D C}_{\mathfrak{p}}$ is not a field, because of the fact that the elements of the form $w=0+z_{2} \varepsilon$ are not invertible. All elements of this form are called zero divisors.

ii) $\quad\left\{\mathbb{D} \mathbb{C}_{\mathfrak{p}}, \oplus, \mathbb{R},+, \cdot \odot\right\}$ is a vector space over $\mathbb{R}$ with addition and scalar multiplication defined in Definition 2.

iii) The set $\{1, J, \varepsilon, J \varepsilon\}$ (or $\{1, \varepsilon, J, \varepsilon J\}$ ) is a basis for $\mathbb{D} \mathbb{C}_{\mathfrak{p}}$, i.e. $\mathbb{D} \mathbb{C}_{\mathfrak{p}}=\operatorname{span}\{1, J, \varepsilon, J \varepsilon\}$. Then, $\operatorname{dim} \mathbb{D} \mathbb{C}_{\mathfrak{p}}=4$.

iv) The elements of the basis $\{1, J, \varepsilon, J \varepsilon\}$ satisfy the properties of the commutative multiplication scheme given in Table 2.

Table 2. Multiplication scheme of dual-generalized complex numbers

\begin{tabular}{|l||l||l||l||l||}
\hline \hline$\Delta$ & 1 & $J$ & $\varepsilon$ & $J \varepsilon$ \\
\hline \hline 1 & 1 & $J$ & $\varepsilon$ & $J \varepsilon$ \\
\hline$J$ & $J$ & $\mathfrak{p}$ & $J \varepsilon$ & $\mathfrak{p} \varepsilon$ \\
\hline$\varepsilon$ & $\varepsilon$ & $J \varepsilon$ & 0 & 0 \\
\hline$J \varepsilon$ & $J \varepsilon$ & $\mathfrak{p} \varepsilon$ & 0 & 0 \\
\hline \hline
\end{tabular}

v) A dual-generalized complex number can be given in terms of its basis elements $\{1, J, \varepsilon, J \varepsilon\}$ (or $\{1, \varepsilon, J, \varepsilon J\})$ by using Table 2 as $w=x_{1}+x_{2} J+y_{1} \varepsilon+y_{2} J \varepsilon$, where $J$ indicates the pure generalized complex unit, $\varepsilon$ is the pure dual unit and $J \varepsilon$ is the dual-generalized complex unit. Moreover, $x_{1}$ is called the real part, $x_{2}$ is called the generalized complex part, $y_{1}$ is called the dual part and $y_{2}$ is called the dual-generalized complex part of $w$. 
vi) $\quad\left\{\mathbb{D C}_{\mathfrak{p}}, \oplus, \mathbb{R},+, \cdot \odot, \Delta\right\}$ is an algebra with operations defined in Definition 2 .

Remark 1. $\forall w \in \mathbb{D} \mathbb{C}_{\mathfrak{p}}$, it can be written that

$w=z_{1}+z_{2} \varepsilon=\left(x_{1}+x_{2} J\right)+\left(y_{1}+y_{2} J\right) \varepsilon=\left(x_{1}+y_{1} \varepsilon\right)+\left(x_{2}+y_{2} \varepsilon\right) J=d_{1}+d_{2} J$,

where $d_{1}, d_{2} \in \mathbb{D}$. Hence the set of generalized complex-dual numbers is denoted by $\mathbb{C}_{\mathfrak{p}} \mathbb{D}$ and defined as:

$\mathbb{C}_{\mathfrak{p}} \mathbb{D}=\left\{w=d_{1}+d_{2} J \mid d_{1}, d_{2} \in \mathbb{D}, J^{2}=\mathfrak{p},-\infty<\mathfrak{p}<\infty\right\}$

So, there is no difference between dual-generalized complex numbers and generalized complex-dual numbers.

Conjugation of a number plays a significant role for its algebraic and geometric properties. For dualgeneralized complex numbers, there are five different conjugations and four modulus in $\mathbb{D} \mathbb{C}_{\mathfrak{p}}$ are as follows:

Definition 3. For any $w=z_{1}+z_{2} \varepsilon \in \mathbb{D} \mathbb{C}_{\mathfrak{p}}$, the conjugations and modules of $w$ are defined as in Table 3 . (Note that, for $z=x_{1}+x_{2} J \in \mathbb{C}_{\mathfrak{p}}, x_{1}$ and $x_{2}$ are called real and imaginary part of $z$ and denoted by $\operatorname{Re}(z)=x_{1}$ and $\operatorname{Im}(z)=x_{2}$, respectively. $\bar{z}=x_{1}-x_{2} J$ indicates conjugate and $|z|^{2}$ indicates the module of $z \in \mathbb{C}_{p},[7]$.)

Table 3. Conjugations and modules of dual-generalized complex numbers

\begin{tabular}{|l|l||}
\hline \hline Generalized complex conjugation & $w^{\dagger_{1}}=\bar{z}_{1}+\bar{z}_{2} \varepsilon$ \\
\hline \hline Generalized complex module & $|w|_{\dagger_{1}}^{2}=w \Delta w^{\dagger_{1}}=\left|z_{1}\right|^{2}+2 \operatorname{Re}\left(z_{1} \bar{z}_{2}\right) \varepsilon \in \mathbb{D}$ \\
\hline \hline Dual conjugation & $w^{\dagger_{2}}=z_{1}-z_{2} \varepsilon$ \\
\hline \hline Dual module & $|w|_{\dagger_{2}}^{2}=w \Delta w^{\dagger_{2}}=z_{1}^{2} \in \mathbb{C}_{\mathfrak{p}}$ \\
\hline \hline Coupled conjugation & $w^{\dagger_{3}}=\bar{z}_{1}-\bar{z}_{2} \varepsilon$ \\
\hline \hline Coupled module & $|w|_{\dagger_{3}}^{2}=w \Delta w^{\dagger_{3}}=\left|z_{1}\right|^{2}-2 \operatorname{Im}\left(z_{1} \bar{z}_{2}\right) J \varepsilon \in \mathbb{D} \mathbb{C}_{\mathfrak{p}}$ \\
\hline \hline $\begin{array}{l}\text { Dual-generalized complex conjugation } \\
(w \text { is not a zero divisor) }\end{array}$ & $w^{\dagger_{4}}=\bar{z}_{1}\left(1-\frac{z_{2}}{z_{1}} \varepsilon\right)$ \\
\hline \hline Dual-generalized complex module & $|w|_{\dagger_{4}}^{2}=w \Delta w^{\dagger_{4}}=\left|z_{1}\right|^{2} \in \mathbb{R}$ \\
\hline \hline Anti-dual conjugation & $w^{\dagger_{5}}=z_{2}-z_{1} \varepsilon$ \\
\hline \hline
\end{tabular}


Proposition 2. Let $w_{1}, w_{2}$ and $w=z_{1}+z_{2} \varepsilon \in \mathbb{D} \mathbb{C}_{\mathfrak{p}}$. Then, the following assertions hold:
i) $\quad\left(w^{\dagger_{k}}\right)^{\dagger_{k}}=w, k=1,2,3,4,\left(z_{1} \neq 0\right.$ for $\left.w^{\dagger_{4}}\right)$,
ii) $\left(w^{\dagger_{5}}\right)^{\dagger_{5}}=-w$,
iii) $\quad\left(w_{1} \oplus w_{2}\right)^{\dagger_{k}}=w_{1}^{\dagger_{k}} \oplus w_{2}^{\dagger_{k}}, k=1,2,3,5$,
iv) $\quad\left(w_{1} \ominus w_{2}\right)^{\dagger_{k}}=w_{1}^{\dagger_{k}} \ominus w_{2}^{\dagger_{k}}, k=1,2,3,5$,
v) $\quad\left(w_{1} \Delta w_{2}\right)^{\dagger_{k}}=w_{1}^{\dagger_{k}} \Delta w_{2}^{\dagger_{k}},\left(z_{1} \neq 0\right.$ for $\left.w^{\dagger_{4}}\right)$,
vi) $\left(\frac{1}{w}\right)^{\dagger_{k}}=\frac{1}{w^{\dagger_{k}}}, k=2,4,\left(z_{1} \neq 0\right.$ for $\left.w^{\dagger_{4}}\right)$,
vii) $\quad w \oplus w^{\dot{\dagger}_{1}}=2 \operatorname{Re}\left(z_{1}\right)+2 \operatorname{Re}\left(z_{2}\right) \varepsilon \in \mathbb{D}$,
viii) $\quad w \oplus w^{\dagger_{2}}=2 z_{1} \in \mathbb{C}_{\mathfrak{p}}$,
ix) $\quad w \oplus w^{\dagger_{3}}=2 \operatorname{Re}\left(z_{1}\right)+2 \operatorname{Im}\left(z_{2}\right) J \varepsilon \in \mathbb{D} \mathbb{C}_{p}$,
x) $z_{1} \cdot w^{\dagger_{4}}=\bar{z}_{1} \cdot w^{\dagger_{2}} \in \mathbb{D} \mathbb{C}_{\mathfrak{p}}$, where $\cdot: \mathbb{C}_{\mathfrak{p}} \times \mathbb{D} \mathbb{C}_{\mathfrak{p}} \rightarrow \mathbb{D} \mathbb{C}_{\mathfrak{p}}$,
xi) $\quad z_{1}=w \ominus\left(w^{\dagger_{5}} \Delta \varepsilon\right), z_{2}=w^{\dagger_{5}} \oplus(w \Delta \varepsilon)$.

Definition 4. Under the condition that $w$ is not a zero divisor, the inverse of any dual-generalized complex number $w$ is defined as

$$
\frac{1}{w}=\frac{w^{\dagger_{2}}}{|w|_{\dagger_{2}}^{2}}=\frac{w^{\dagger_{4}}}{|w|_{\dagger_{4}}^{2}} \text {. }
$$

\subsection{Dual-Generalized Complex Valued Functions}

Here, we examine the dual-generalized complex valued function. In order to give this examination, we firstly give the following definition:

Definition 5. The following statements can be given for the set $\mathbb{D C}_{\mathfrak{p}}$ :

i) $\Omega$ is called a dual-generalized complex subset of $\mathbb{D} \mathbb{C}_{\mathfrak{p}}$ if there exists a subset $S \subset \mathbb{C}_{\mathfrak{p}}$ such that $\Omega \approx S \times \mathbb{C}_{\mathfrak{p}}$. Here $S$ is called generator of $\Omega$.

ii) $\Omega$ is an open dual-generalized complex subset of $\mathbb{D C}_{\mathfrak{p}}$ if the generator of $\Omega$ is an open subset of $\mathbb{C}_{p}$.

iii) $\Omega$ is a closed dual-generalized complex subset of $\mathbb{D C}_{\mathfrak{p}}$ if its complement is an open subset of $\mathbb{D C} \mathbb{C}_{p}$.

Definition 6. A dual-generalized complex valued function $f$ is a mapping such that:

$$
\begin{aligned}
f: \Omega \subset \mathbb{D C}_{\mathfrak{p}} & \rightarrow \mathbb{D C}_{\mathfrak{p}} \\
z_{1}+z_{2} \varepsilon & \rightarrow f\left(z_{1}+z_{2} \varepsilon\right),
\end{aligned}
$$

where $\Omega$ is an open dual-generalized complex subset of $\mathbb{D C}_{\mathfrak{p}}$. 
Definition 7. The dual-generalized complex valued function $f$ is called continuous at $w_{0}=z_{01}+z_{02} \varepsilon$ if $\lim _{w \rightarrow w_{0}} f(w)=f\left(w_{0}\right)$. Moreover, $f$ is called continuous in open subset $\Omega \subset \mathbb{D} \mathbb{C}_{\mathfrak{p}}$ if it is continuous at every point of $\Omega$.

Definition 8. The dual-generalized complex valued function $f$ is differentiable at $w_{0}=z_{01}+z_{02} \varepsilon$ if the following limit exits:

$$
\frac{d f}{d w}\left(w_{0}\right)=\lim _{w \rightarrow w_{0}} \frac{f(w)-f\left(w_{0}\right)}{w-w_{0}} .
$$

$\frac{d f}{d w}\left(w_{0}\right)$ is called the derivative of $f$ at the point $w_{0}$. Besides, $f$ is differentiable in an open subset $\Omega \subset \mathbb{D C}_{\mathfrak{p}}$ if it is differentiable at every point of $\Omega$.

Theorem 1. Let $f$ be an dual-generalized complex infinitely differentiable function in open subset $\Omega \subset \mathbb{D C}_{\mathfrak{p}}$. For every $w=z_{1}+z_{2} \varepsilon \in \Omega$, it can be written as:

$f\left(z_{1}+z_{2} \varepsilon\right)=f\left(z_{1}\right)+z_{2} f^{\prime}\left(z_{1}\right) \varepsilon$.

Proof. The Taylor series of $f\left(z_{1}+z_{2} \varepsilon\right)$ at $w=0$ can be written as:

$$
f(w)=f(0)+f^{\prime}(0)\left(z_{1}+z_{2} \varepsilon\right)+f^{\prime \prime}(0) \frac{z_{1}^{2}+2 z_{1} z_{2} \varepsilon}{2 !}+\ldots+f^{(k)}(0) \frac{z_{1}^{k}+\left(k z_{1}^{k-1} z_{2}\right) \varepsilon}{k !}+\ldots
$$

If we rearrange the above equation, we get

$$
f(w)=\left(f(0)+f^{\prime}(0) z_{1}+f^{\prime \prime}(0) \frac{z_{1}^{2}}{2 !}+\ldots+f^{(k)}(0) \frac{z_{1}^{k}}{k !}+\ldots\right)+z_{2}\left(f^{\prime}(0)+f^{\prime \prime}(0) z_{1}+\ldots+f^{(k)}(0) \frac{z_{1}^{k-1}}{(k-1) !}+\ldots\right) \varepsilon .
$$

Then, we have $f(w)=f\left(z_{1}\right)+z_{2} f^{\prime}\left(z_{1}\right) \varepsilon$.

Example 1. Some basic examples for the dual-generalized complex valued functions are given as follows:
i) $e^{z_{1}+z_{2} \varepsilon}=e^{z_{1}}+z_{2} e^{z_{1}} \varepsilon$,
ii) $\sqrt{z_{1}+z_{2} \varepsilon}=\sqrt{z_{1}}+z_{2} \frac{1}{2 \sqrt{z_{1}}} \varepsilon$,
iii) $\ln \left(z_{1}+z_{2} \varepsilon\right)=\ln z_{1}+\frac{z_{2}}{z_{1}} \varepsilon$,
iv) $\quad\left(z_{1}+z_{2} \varepsilon\right)^{n}=z_{1}^{n}+n z_{2} z_{1}^{n-1} \varepsilon$.

\section{2. Matrix Representations of Dual-Generalized Complex Numbers}

Here, we first define the following transformation

$$
\begin{aligned}
f: \mathbb{D C}_{\mathfrak{p}} & \rightarrow \mathbb{D C}_{\mathfrak{p}} \\
w_{1} & \rightarrow f_{w}\left(w_{1}\right)=w \Delta w_{1},
\end{aligned}
$$


for every $w=z_{1}+z_{2} \varepsilon=x_{1}+x_{2} J+y_{1} \varepsilon+y_{2} J \varepsilon \in \mathbb{D C}_{\mathfrak{p}}$. A trivial computation shows that $f$ is a linear transformation. The real and generalized complex matrix representations of $f$ (of $w$ as well) with respect to the basis $\{1, J, \varepsilon, J \varepsilon\}$ and $\{1, \varepsilon\}$ (basis for $\mathbb{D}$ ) are respectively given as follows:

$$
A_{\mathbb{R}}=\left[\begin{array}{cccc}
x_{1} & \mathfrak{p} x_{2} & 0 & 0 \\
x_{2} & x_{1} & 0 & 0 \\
y_{1} & \mathfrak{p} y_{2} & x_{1} & \mathfrak{p} x_{2} \\
y_{2} & y_{1} & x_{2} & x_{1}
\end{array}\right], A_{\mathbb{C}_{\mathfrak{p}}}=\left[\begin{array}{cc}
x_{1}+x_{2} J & 0 \\
y_{1}+y_{2} J & x_{1}+x_{2} J
\end{array}\right]=\left[\begin{array}{cc}
z_{1} & 0 \\
z_{2} & z_{1}
\end{array}\right] .
$$

Moreover, $\operatorname{det}\left(A_{\mathbb{C}_{p}}\right)=z_{1}^{2}=|w|_{\dot{t}_{2}}^{2}$.

There is no loss of generality in assuming that $w=z_{1}+z_{2} \varepsilon=d_{1}+d_{2} J \in \mathbb{C}_{\mathfrak{p}} \mathbb{D}$. Similarly, we can write the matrices of $w$ with respect to the basis $\{1, \varepsilon, J, \varepsilon J\}$ and $\{1, J\}$, respectively:

$$
B_{\mathbb{R}}=\left[\begin{array}{cccc}
x_{1} & 0 & \mathfrak{p} x_{2} & 0 \\
y_{1} & x_{1} & \mathfrak{p} y_{2} & \mathfrak{p} x_{2} \\
x_{2} & 0 & x_{1} & 0 \\
y_{2} & x_{2} & y_{1} & x_{1}
\end{array}\right], B_{\mathbb{D}}=\left[\begin{array}{cc}
x_{1}+y_{1} \varepsilon & \mathfrak{p}\left(x_{2}+y_{2} \varepsilon\right) \\
x_{2}+y_{2} \varepsilon & x_{1}+y_{1} \varepsilon
\end{array}\right]=\left[\begin{array}{cc}
d_{1} & \mathfrak{p} d_{2} \\
d_{2} & d_{1}
\end{array}\right] .
$$

Besides, $\operatorname{det}\left(B_{\mathbb{D}}\right)=d_{1}^{2}-\mathfrak{p} d_{2}^{2}=|w|_{\dot{t}_{1}}^{2}$.

Example 2. The matrix representations of the dual-generalized complex number $w=-2+5 J+\varepsilon-4 J \varepsilon$ can be calculated with respect to basis $\{1, J, \varepsilon, J \varepsilon\}$ and $\{1, \varepsilon\}$, respectively as follows:

$$
A_{\mathbb{R}}=\left[\begin{array}{cccc}
-2 & 5 \mathfrak{p} & 0 & 0 \\
5 & -2 & 0 & 0 \\
1 & -4 \mathfrak{p} & -2 & 5 \mathfrak{p} \\
-4 & 1 & 5 & -2
\end{array}\right], A_{\mathbb{C}_{\mathfrak{p}}}=\left[\begin{array}{cc}
-2+5 J & 0 \\
1-4 J & -2+5 J
\end{array}\right]
$$

and with respect to basis $\{1, \varepsilon, J, \varepsilon J\}$ and $\{1, J\}$, respectively as follows:

$$
B_{\mathbb{R}}=\left[\begin{array}{cccc}
-2 & 0 & 5 \mathfrak{p} & 0 \\
1 & -2 & -4 \mathfrak{p} & 5 \mathfrak{p} \\
5 & 0 & -2 & 0 \\
-4 & 5 & 1 & -2
\end{array}\right], B_{\mathbb{D}}=\left[\begin{array}{cc}
-2+\varepsilon & \mathfrak{p}(5-4 \varepsilon) \\
5-4 \varepsilon & -2+\varepsilon
\end{array}\right]
$$

Also, $\operatorname{det}\left(A_{\mathbb{C}_{\mathfrak{p}}}\right)=(4+25 \mathfrak{p})-20 J$ and $\operatorname{det}\left(B_{\mathbb{D}}\right)=(4-25 \mathfrak{p})+(-4+40 \mathfrak{p}) \varepsilon$ 


\section{HYPERBOLIC-GENERALIZED COMPLEX NUMBERS}

In this original section, we present the concept of hyperbolic-generalized complex numbers.

Definition 9. A hyperbolic-generalized complex number $\tilde{w}$ is an ordered pair of generalized complex numbers $\left(z_{1}, z_{2}\right)$ and denoted in the form

$$
\tilde{w}=\left(z_{1}, z_{2}\right)=z_{1}+z_{2} j
$$

where $z_{1}=x_{1}+x_{2} J \in \mathbb{C}_{\mathfrak{p}}, z_{2}=y_{1}+y_{2} J \in \mathbb{C}_{\mathfrak{p}}, J^{2}=\mathfrak{p},-\infty<\mathfrak{p}<\infty$ and $j^{2}=1, j \neq \pm 1$. This special number is a hyperbolic number with generalized complex coefficients. The set of these numbers is denoted by $\mathbb{H} \mathbb{C}_{\mathfrak{p}}$ and defined as

$\mathbb{H} \mathbb{C}_{\mathfrak{p}}=\left\{\tilde{w}=z_{1}+z_{2} j \mid z_{1}, z_{2} \in \mathbb{C}_{\mathfrak{p}}, j^{2}=1 j \neq \pm 1\right\}$.

If all values are non-zero, $\tilde{w}$ is called a proper hyperbolic-generalized complex number.

Definition 10. Let $\tilde{w}_{1}, \tilde{w}_{2} \in \mathbb{H} \mathbb{C}_{\mathfrak{p}}$ and $\lambda \in \mathbb{R}$. Then, the addition, substraction, multiplication, scalar multiplication and equality of hyperbolic-generalized complex numbers are defined respectively as in the following table:

Table 4. Operations for hyperbolic-generalized complex numbers

\begin{tabular}{|l||r||c|}
\hline Addition & $\begin{array}{r}\oplus: \mathbb{H} \mathbb{C}_{\mathfrak{p}} \times \mathbb{H} \mathbb{C}_{\mathfrak{p}} \rightarrow \mathbb{H} \mathbb{C}_{\mathfrak{p}} \\
\left(\tilde{w}_{1}, \tilde{w}_{2}\right) \rightarrow \tilde{w}_{1} \oplus \tilde{w}_{2}\end{array}$ & $\begin{array}{r}\tilde{w}_{1} \oplus \tilde{w}_{2}=\left(z_{11}+z_{12} j\right) \oplus\left(z_{21}+z_{22} j\right) \\
=\left(z_{11}+z_{21}\right)+\left(z_{12}+z_{22}\right) j\end{array}$ \\
\hline \hline Substraction & $\begin{array}{r}\ominus: \mathbb{H} \mathbb{C}_{\mathfrak{p}} \times \mathbb{H} \mathbb{C}_{\mathfrak{p}} \rightarrow \mathbb{H} \mathbb{C}_{\mathfrak{p}} \\
\left(\tilde{w}_{1}, \tilde{w}_{2}\right) \rightarrow \tilde{w}_{1} \odot \tilde{w}_{2}\end{array}$ & $\begin{array}{r}\tilde{w}_{1} \odot \tilde{w}_{2}=\left(z_{11}+z_{12} j\right) \odot\left(z_{21}+z_{22} j\right) \\
=\left(z_{11}-z_{21}\right)+\left(z_{12}-z_{22}\right) j\end{array}$ \\
\hline Multiplication & $\begin{array}{r}\Delta: \mathbb{H} \mathbb{C}_{\mathfrak{p}} \times \mathbb{H} \mathbb{C}_{\mathfrak{p}} \rightarrow \mathbb{H} \mathbb{C}_{\mathfrak{p}} \\
\left(\tilde{w}_{1}, \tilde{w}_{2}\right) \rightarrow \tilde{w}_{1} \Delta \tilde{w}_{2}\end{array}$ & $\begin{array}{r}\tilde{w}_{1} \Delta \tilde{w}_{2}=\left(z_{11}+z_{12} j\right) \Delta\left(z_{21}+z_{22} j\right) \\
=\left(z_{11} z_{21}+z_{12} z_{22}\right)+\left(z_{11} z_{22}+z_{12} z_{21}\right) j\end{array}$ \\
\hline \hline Scalar Multiplication & $\begin{array}{r}\odot: \mathbb{R} \times \mathbb{H} \mathbb{C}_{\mathfrak{p}} \rightarrow \mathbb{H} \mathbb{C}_{\mathfrak{p}} \\
\left(\lambda, \tilde{w}_{1}\right) \rightarrow \lambda \odot \tilde{w}_{1}\end{array}$ & $\begin{array}{r}\lambda \odot \tilde{w}_{1}=\lambda \odot\left(z_{11}+z_{12} j\right) \\
=\left(\lambda z_{11}\right)+\left(\lambda z_{12}\right) j\end{array}$ \\
\hline Equality & $\tilde{w}_{1}=\tilde{w}_{2} \Leftrightarrow z_{11}+z_{12} j=z_{21}+z_{22} j \Leftrightarrow z_{11}=z_{21} \wedge z_{12}=z_{22}$ \\
\hline
\end{tabular}

Proposition 3. The algebraic properties of the set of hyperbolic-generalized complex numbers $\mathbb{H} \mathbb{C}_{\mathfrak{p}}$ are given as follows:

i) $\left(\mathbb{H C}_{\mathfrak{p}}, \oplus, \Delta\right)$ is a commutative ring with unity under addition and multiplication as defined in Definition 10. The additive and multiplicative identities of this ring are $(0,0)=0+0 j$ and $(1,0)=1+0 j$, respectively. But, $\mathbb{H} \mathbb{C}_{\mathfrak{p}}$ is not a field, owing to the fact that the elements of the form $\tilde{w}=z_{1}+z_{2} j$ are not invertible when $z_{1}^{2}-z_{2}^{2}=0$. All elements of this form are called zero divisors.

ii) $\quad\left\{\mathbb{H}_{\mathfrak{p}}, \oplus, \mathbb{R},+, \cdot, \odot\right\}$ is a vector space over $\mathbb{R}$ with addition and scalar multiplication defined in Definition 10. 
iii) The set $\{1, J, j, J j\}$ (or $\{1, j, J, j J\}$ ) is a basis for $\mathbb{H C}_{\mathfrak{p}}$, i.e. $\mathbb{H}_{\mathfrak{p}}=s p\{1, J, j, J j\}$. Then $\operatorname{dim} \mathbb{H} \mathbb{C}_{\mathfrak{p}}=4$.

iv) The elements of the basis $\{1, J, j, J j\}$ satisfy the properties of the following commutative multiplication scheme given in Table 5 .

Table 5. Multiplication scheme of hyperbolic-generalized complex numbers

\begin{tabular}{|l||l||l||l||l||}
\hline$\Delta$ & 1 & $J$ & $j$ & $J j$ \\
\hline \hline 1 & 1 & $J$ & $j$ & $J j$ \\
\hline$J$ & $J$ & $\mathfrak{p}$ & $J j$ & $\mathfrak{p} j$ \\
\hline \hline$j$ & $j$ & $J j$ & 1 & $J$ \\
\hline$J j$ & $J j$ & $\mathfrak{p} j$ & $J$ & $\mathfrak{p}$ \\
\hline
\end{tabular}

v) A hyperbolic-generalized complex number can be given in terms of its basis elements $\{1, J, j, J j\}$ (or $\{1, j, J, j J\}$ ) by using Table 5 as $\tilde{w}=x_{1}+x_{2} J+y_{1} j+y_{2} J j$, where $J$ indicates the pure generalized complex unit, $j$ is the pure hyperbolic unit and $J j$ is the hyperbolic- generalized complex unit. Besides, $x_{1}$ is called the real part, $x_{2}$ is called the generalized complex part, $y_{1}$ is called the hyperbolic part and $y_{2}$ is called the hyperbolic-generalized complex part of $\tilde{w}$.

vi) $\quad\left\{\mathbb{H} \mathbb{C}_{\mathfrak{p}}, \oplus, \mathbb{R},+, \cdot, \odot, \Delta\right\}$ is an algebra with operations defined in Definition 10 .

Remark 2. $\forall \tilde{w} \in \mathbb{H} \mathbb{C}_{\mathfrak{p}}$, the following equation can be written:

$$
\tilde{w}=z_{1}+z_{2} j=\left(x_{1}+x_{2} J\right)+\left(y_{1}+J y_{2}\right) j=\left(x_{1}+y_{1} j\right)+\left(x_{2}+y_{2} j\right) J=h_{1}+h_{2} J,
$$

where $h_{1}, h_{2} \in \mathbb{H}$. Then the set of generalized complex-hyperbolic numbers is denoted by $\mathbb{C}_{\mathfrak{p}} \mathbb{H}$ and defined as:

$$
\mathbb{C}_{\mathfrak{p}} \mathbb{H}=\left\{\tilde{w}=h_{1}+h_{2} J \mid h_{1}, h_{2} \in \mathbb{H}, J^{2}=\mathfrak{p},-\infty<\mathfrak{p}<\infty\right\} .
$$

Hence, there exists no difference between hyperbolic-generalized complex numbers and generalized complex-hyperbolic numbers.

On the other hand, every hyperbolic number can be written in terms of the basis $\{1, j\}$. Since the hyperbolic numbers have idempotent basis (see in [2]), we can express the hyperbolic-generalized complex number in terms of the elements of the set $\left\{j_{+}=\frac{1+j}{2}, j_{-}=\frac{1-j}{2}\right\}$ as follows:

$$
\begin{aligned}
\tilde{w}=z_{1}+z_{2} j & =\left(x_{1}+x_{2} J\right)+\left(y_{1}+y_{2} J\right) j \\
& =\left[\left(x_{1}+y_{1}\right)+\left(x_{2}+y_{2}\right) J\right]\left(\frac{1+j}{2}\right)+\left[\left(x_{1}-y_{1}\right)+\left(x_{2}-y_{2}\right) J\right]\left(\frac{1-j}{2}\right) \\
& =z_{+} j_{+}+z_{-} j_{-},
\end{aligned}
$$

where $z_{+}=\left(x_{1}+y_{1}\right)+\left(x_{2}+y_{2}\right) J$ and $z_{-}=\left(x_{1}-y_{1}\right)+\left(x_{2}-y_{2}\right) J$. 
Definition 11. The set $\left\{j_{+}=\frac{1+j}{2}, j_{-}=\frac{1-j}{2}\right\}$ is called an idempotent basis for hyperbolic-generalized complex numbers similar to hyperbolic numbers. Because of the fact that $j_{+}^{2}=j_{+}, j_{-}^{2}=j_{-}$and they are mutually annihilating because $j_{+} j_{-}=0$.

Definition 12. $\forall \tilde{w}=z_{1}+z_{2} j \in \mathbb{H} \mathbb{C}_{\mathfrak{p}}$, the conjugations and modules of $\tilde{w}$ are defined as in Table 6 .

Table 6. Conjugations and modules of hyperbolic-generalized complex numbers

\begin{tabular}{|c|c|}
\hline Generalized complex conjugation & $\tilde{w}^{\dagger_{1}}=\bar{z}_{1}+\bar{z}_{2} j$ \\
\hline Generalized complex module & $|\tilde{w}|_{\dagger_{1}}^{2}=\tilde{w} \Delta \tilde{w}^{\dagger_{1}}=\left|z_{1}\right|^{2}+\left|z_{2}\right|^{2}+2 \operatorname{Re}\left(z_{1} \bar{z}_{2}\right) j \in \mathbb{H}$ \\
\hline Hyperbolic conjugation & $\tilde{w}^{\dagger_{2}}=z_{1}-z_{2} j$ \\
\hline Hyperbolic module & $|\tilde{w}|_{\dot{\dagger}_{2}}^{2}=\tilde{w} \Delta \tilde{w}^{\dagger_{2}}=z_{1}^{2}-z_{2}^{2} \in \mathbb{C}_{p}$ \\
\hline Coupled conjugation & $\tilde{w}^{\dagger_{3}}=\bar{z}_{1}-\bar{z}_{2} j$ \\
\hline Coupled module & $\left.\left|\tilde{w}_{\dot{+}_{3}}^{2}=\tilde{w} \Delta \tilde{w}^{\dagger_{3}}=\right| z_{1}\right|^{2}-\left|z_{2}\right|^{2}-2 \operatorname{Im}\left(z_{1} \bar{z}_{2}\right) J j \in \mathbb{H} \mathbb{C}_{p}$ \\
\hline Hyperbolic-generalized complex conjugation & $\tilde{w}^{\dagger_{4}}=\bar{z}_{1}\left(1-\frac{z_{2}}{z_{1}} j\right)$ \\
\hline Hyperbolic-generalized complex module & $|\tilde{w}|_{\grave{\dagger}_{4}}^{2}=\tilde{w} \Delta \tilde{w}^{\dagger_{4}}=\left|z_{1}\right|^{2}-\frac{\bar{z}_{1} z_{2}^{2}}{z_{1}}$ \\
\hline Anti-hyperbolic conjugation & $\tilde{w}^{\dagger_{s}}=z_{2}-z_{1} j$ \\
\hline Anti-hyperbolic module & $|\tilde{w}|_{\dot{\dagger}_{s}}^{2}=\tilde{w} \Delta \tilde{w}^{\dagger_{s}}=\left(z_{2}^{2}-z_{1}^{2}\right) j \in \mathbb{H} \mathbb{C}_{\mathfrak{p}}$ \\
\hline
\end{tabular}

Proposition 4. Let $\tilde{w}_{1}, \tilde{w}_{2}$ and $\tilde{w}=z_{1}+j z_{2} \in \mathbb{H} \mathbb{C}_{\mathfrak{p}}$. Then, the following assertions hold:
i) $\quad\left(\tilde{w}^{\dagger_{k}}\right)^{\dagger_{k}}=\tilde{w}, \quad k=1,2,3,4$,
ii) $\left(\tilde{w}^{\dagger_{5}}\right)^{\dagger_{5}}=-\tilde{w}$,
iii) $\quad\left(\tilde{w}_{1} \oplus \tilde{w}_{2}\right)^{\dagger_{k}}=\tilde{w}_{1}^{\dagger_{k}} \oplus \tilde{w}_{2}^{\dagger_{k}}, k=1,2,3,4,5$,
iv) $\quad\left(\tilde{w}_{1} \ominus \tilde{w}_{2}\right)^{\dagger_{k}}=\tilde{w}_{1}^{\dagger_{k}} \ominus \tilde{w}_{2}^{\dagger_{k}}, k=1,2,3,4,5$,
v) $\quad\left(\tilde{w}_{1} \Delta \tilde{w}_{2}\right)^{\dagger_{k}}=\tilde{w}_{1}^{\dagger_{k}} \Delta \tilde{w}_{2}^{\dagger_{k}}, k=1,2,3$,
vi) $\left(\frac{1}{\tilde{w}}\right)^{\dagger_{k}}=\frac{1}{\tilde{w}^{\dagger_{k}}},(\tilde{w}$ is not zero divisor $), k=1,2$,
vii) $\tilde{w} \oplus \tilde{w}^{\dagger_{1}}=2 \operatorname{Re}\left(z_{1}\right)+j 2 \operatorname{Re}\left(z_{2}\right) \in \mathbb{H}$,
viii) $\tilde{w} \oplus \tilde{w}^{\dagger_{2}}=2 z_{1} \in \mathbb{C}_{\mathfrak{p}}$,
ix) $\quad \tilde{w} \oplus \tilde{w}^{\dagger_{3}}=2 \operatorname{Re}\left(z_{1}\right)+2 j J \operatorname{Im}\left(z_{2}\right) \in \mathbb{H} \mathbb{C}_{p}$,
x) $\quad z_{1} \cdot \tilde{w}^{\dagger_{4}}=\bar{z}_{1} \cdot \tilde{w}^{\dagger_{2}} \in \mathbb{H} \mathbb{C}_{\mathfrak{p}}$, where $\cdot: \mathbb{C}_{\mathfrak{p}} \times \mathbb{H} \mathbb{C}_{\mathfrak{p}} \rightarrow \mathbb{H} \mathbb{C}_{\mathfrak{p}}$,
xi) $\quad 2 z_{1}=\tilde{w} \Theta\left(\tilde{w}^{\dagger_{s}} \Delta j\right), 2 z_{2}=\tilde{w}^{\dagger_{5}} \oplus(\tilde{w} \Delta j)$. 
Definition 13. Under the condition that $\tilde{w}$ is not a zero divisor, the inverse of $\tilde{w} \in \mathbb{H} \mathbb{C}_{\mathfrak{p}}$ is defined as $\frac{1}{\tilde{w}}=\frac{\tilde{w}^{\dagger_{1}}}{|\tilde{w}|_{\dagger_{1}}^{2}}=\frac{\tilde{w}^{\dagger_{2}}}{|\tilde{w}|_{\dagger_{2}}^{2}}$

\section{1. Hyperbolic-Generalized Complex Valued Functions}

Similar to Section 2.1, here we first define hyperbolic-generalized complex open subset $\Omega \subset \mathbb{H} \mathbb{C}_{\mathfrak{p}}$ and the mapping as follows:

Definition 14. The following statements can be given for the set $\mathbb{H}_{\mathbb{C}_{p}}$ :

$$
\begin{aligned}
f: \Omega \subset \mathbb{H} \mathbb{C}_{\mathfrak{p}} & \rightarrow \mathbb{H} \mathbb{C}_{\mathfrak{p}} \\
\left(z_{1}+z_{2} j\right) & \rightarrow f\left(z_{1}+z_{2} j\right) .
\end{aligned}
$$

Then, it can be written by using idempotent basis as follows:

$f(\tilde{w})=f\left(z_{+} j_{+}+z_{-} j_{-}\right)=f\left(z_{+}\right) j_{+}+f\left(z_{-}\right) j_{-}$.

Definition 15. The hyperbolic-generalized complex valued function $f$ is called continuous at $\tilde{w}_{0}=z_{01}+z_{02} j$ if $\lim _{\tilde{w} \rightarrow \tilde{w}_{0}} f(\tilde{w})=f\left(\tilde{w}_{0}\right)$. Moreover, $f$ is called continuous in open subset $\Omega \subset \mathbb{H} \mathbb{C}_{\mathfrak{p}}$ if it is continuous at every point of $\Omega$.

Definition 16. The hyperbolic-generalized complex valued function $f$ is differentiable at $\tilde{w}_{0}=z_{01}+z_{02} j$ if the following limit exits:

$\frac{d f}{d \tilde{w}}\left(\tilde{w}_{0}\right)=\lim _{\tilde{w} \rightarrow \tilde{w}_{0}} \frac{f(\tilde{w})-f\left(\tilde{w}_{0}\right)}{\tilde{w}-\tilde{w}_{0}}$.

$\frac{d f}{d \tilde{w}}\left(\tilde{w}_{0}\right)$ is called the derivative of $f$ at the point $\tilde{w}_{0}$. Besides, $f$ is differentiable in an open subset $\Omega \subset \mathbb{H} \mathbb{C}_{\mathfrak{p}}$ if it is differentiable at every point of $\Omega$.

Example 3. For $\tilde{w}=z_{1}+z_{2} j \in \mathbb{H C}_{\mathfrak{p}}$, some hyperbolic-generalized complex valued functions are given as follows:
i) $\quad\|\tilde{w}\|^{2}=\frac{1}{2}\left[\left\|z_{1}+z_{2}\right\|^{2}+\left\|z_{1}-z_{2}\right\|^{2}\right]+\frac{1}{2}\left[\left\|z_{1}+z_{2}\right\|^{2}-\left\|z_{1}-z_{2}\right\|^{2}\right] j$,
ii) $\quad e^{z_{1}+z_{2}} j=\frac{1}{2}\left(e^{z_{1}+z_{2}}+e^{z_{1}-z_{2}}\right)+\frac{1}{2}\left(e^{z_{1}+z_{2}}-e^{z_{1}-z_{2}}\right) j$,
iii) $\quad\left|z_{1}+z_{2} j\right|=\frac{1}{2}\left(\left|z_{1}+z_{2}\right|+\left|z_{1}-z_{2}\right|\right)+\frac{1}{2}\left(\left|z_{1}+z_{2}\right|-\left|z_{1}-z_{2}\right|\right) j$,
iv) $\ln \left(z_{1}+z_{2} j\right)=\frac{1}{2} \ln \left(z_{1}^{2}-z_{2}^{2}\right)+\frac{1}{2} \ln \left(\frac{z_{1}+z_{2}}{z_{1}-z_{2}}\right) j,\left(z_{1}^{2}-z_{2}^{2}\right)>0,\left(z_{1}-z_{2}\right) \neq 0,\left(\frac{z_{1}+z_{2}}{z_{1}-z_{2}}\right)>0$,
v) $\left(z_{1}+z_{2} j\right)^{n}=\left(z_{1}+z_{2}\right)^{n}\left(\frac{1+j}{2}\right)+\left(z_{1}-z_{2}\right)^{n}\left(\frac{1-j}{2}\right)$. 


\section{2. Matrix Representations of Hyperbolic-Generalized Complex Numbers}

Here, we first define the transformation $f: \mathbb{H}_{\mathfrak{p}} \rightarrow \mathbb{H}_{\mathfrak{p}}, f_{\tilde{w}}(\tilde{w})=\tilde{w} \Delta \tilde{w}_{1}$, for every $\tilde{w}=z_{1}+z_{2} j=x_{1}+x_{2} J+y_{1} j+y_{2} J j$. It is obvious that $f$ is linear. The real and generalized complex matrix representations of $f$ (of $\tilde{w}$ as well ) with respect to the basis $\{1, J, j, J j\}$ and $\{1, j\}$ (basis for $\mathbb{H}$ ) can be given as below, respectively:

$\tilde{A}_{\mathbb{R}}=\left[\begin{array}{cccc}x_{1} & \mathfrak{p} x_{2} & y_{1} & \mathfrak{p} y_{2} \\ x_{2} & x_{1} & y_{2} & y_{1} \\ y_{1} & \mathfrak{p} y_{2} & x_{1} & \mathfrak{p} x_{2} \\ y_{2} & y_{1} & x_{2} & x_{1}\end{array}\right], \tilde{A}_{\mathbb{C}_{\mathfrak{p}}}=\left[\begin{array}{cc}x_{1}+J x_{2} & y_{1}+J y_{2} \\ y_{1}+J y_{2} & x_{1}+J x_{2}\end{array}\right]=\left[\begin{array}{cc}z_{1} & z_{2} \\ z_{2} & z_{1}\end{array}\right]$,

where $\operatorname{det}\left(\tilde{A}_{\mathbb{C}_{\mathfrak{p}}}\right)=z_{1}^{2}-z_{2}^{2}=|\tilde{w}|_{\grave{t}_{2}}^{2}$

By writing $\tilde{w}=z_{1}+z_{2} j=h_{1}+h_{2} J \in \mathbb{C}_{\mathfrak{p}} \mathbb{H}$, the matrices of $\tilde{w}$ with respect to the basis $\{1, j, J, j J\}$ and $\{1, J\}$ are given respectively as follows:

$\tilde{B}_{\mathbb{R}}=\left[\begin{array}{cccc}x_{1} & y_{1} & \mathfrak{p} x_{2} & \mathfrak{p} y_{2} \\ y_{1} & x_{1} & \mathfrak{p} y_{2} & \mathfrak{p} x_{2} \\ x_{2} & y_{2} & x_{1} & y_{1} \\ y_{2} & x_{2} & y_{1} & x_{1}\end{array}\right], \tilde{B}_{\mathbb{H}}=\left[\begin{array}{cc}x_{1}+j y_{1} & \mathfrak{p}\left(x_{2}+j y_{2}\right) \\ x_{2}+j y_{2} & x_{1}+j y_{1}\end{array}\right]=\left[\begin{array}{cc}h_{1} & \mathfrak{p} h_{2} \\ h_{2} & h_{1}\end{array}\right]$,

where $\operatorname{det}\left(\tilde{B}_{\mathbb{H}}\right)=h_{1}^{2}-\mathfrak{p} h_{2}^{2}=|\tilde{w}|_{\dot{t}_{1}}^{2}$.

Example 4. The matrix representations of $\tilde{w}=-2+5 J+j-4 J j$ with respect to the basis $\{1, J, j, J j\}$ and $\{1, j\}$ can be given as below, respectively:

$$
\tilde{A}_{\mathbb{R}}=\left[\begin{array}{cccc}
-2 & 5 \mathfrak{p} & 1 & -4 \mathfrak{p} \\
5 & -2 & -4 & 1 \\
1 & -4 \mathfrak{p} & -2 & 5 \mathfrak{p} \\
-4 & 1 & 5 & -2
\end{array}\right], \tilde{A}_{\mathbb{C}_{\mathfrak{p}}}=\left[\begin{array}{cc}
-2+5 J & 1-4 J \\
1-4 J & -2+5 J
\end{array}\right],
$$

and with respect to the basis $\{1, j, J, j J\}$ and $\{1, J\}$ respectively:

$$
\tilde{B}_{\mathbb{R}}=\left[\begin{array}{cccc}
-2 & 1 & 5 \mathfrak{p} & -4 \mathfrak{p} \\
1 & -2 & -4 \mathfrak{p} & 5 \mathfrak{p} \\
5 & -4 & -2 & 1 \\
-4 & 5 & 1 & -2
\end{array}\right], \tilde{B}_{\mathbb{H}}=\left[\begin{array}{cc}
-2+j & \mathfrak{p}(5-4 j) \\
5-4 j & -2+j
\end{array}\right] .
$$

Moreover, $\operatorname{det}\left(\tilde{A}_{\mathbb{C}_{\mathfrak{p}}}\right)=(3+9 \mathfrak{p})-12 J \in \mathbb{C}_{\mathfrak{p}}$ and $\operatorname{det}\left(\tilde{B}_{\mathbb{H}}\right)=(5-41 \mathfrak{p})+(-4+40 \mathfrak{p}) j \in \mathbb{H}$ 


\section{CONCLUSIONS}

In this study, inspired from the theory of the number systems constructed by writing the coefficients as elements of the sets $\mathbb{C}, \mathbb{H}$ and $\mathbb{D}$, we present the theories of dual-generalized complex and hyperbolicgeneralized complex numbers for $\mathfrak{p} \in \mathbb{R}$. By using similar thought, the expressions given in this paper can be given for the set of complex-generalized complex numbers:

$$
\mathbb{C}_{\mathfrak{p}}=\left\{\tilde{\tilde{w}}=z_{1}+z_{2} i \mid z_{1}=x_{1}+x_{2} J, z_{2}=y_{1}+y_{2} J \in \mathbb{C}_{\mathfrak{p}}, J^{2}=\mathfrak{p}, i^{2}=-1\right\} .
$$

If the sets $\mathbb{D} \mathbb{C}_{\mathfrak{p}}, \mathbb{H} \mathbb{C}_{\mathfrak{p}}$ and $\mathbb{C C}_{\mathfrak{p}}$ are examined for special values of $\mathfrak{p} \in\{-1,0,1\}$, then the classification in Table 7 can be given.

In the future studies, it is planned to adapt the theory undertaken in this paper to hybrid numbers created with any combination of the complex, hyperbolic and dual numbers, [31].

Table 7. The examination of the sets $\mathbb{D} \mathbb{C}_{\mathfrak{p}}, \mathbb{H} \mathbb{C}_{\mathfrak{p}}$ and $\mathbb{C}_{\mathfrak{p}}$ for $\mathfrak{p} \in\{-1,0,1\}$

\begin{tabular}{|c|c|c|c|c|}
\hline \multicolumn{3}{|c|}{$i^{2}=-1, \mathbf{i}^{2}=-1$} & & \\
\hline \multirow{3}{*}{$\mathbb{D C} \mathbb{C}_{\mathfrak{p}}$} & \multirow{3}{*}{ 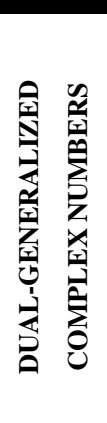 } & \multirow{3}{*}{ 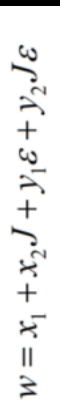 } & $\begin{array}{l}\text { Dual-complex numbers } \\
(\text { see in }[15,16,17])\end{array}$ & $\begin{array}{c}w=x_{1}+x_{2} \mathbf{i}+y_{1} \varepsilon+y_{2} \mathbf{i} \varepsilon \\
J=\mathbf{i}, \mathfrak{p}=-1\end{array}$ \\
\hline & & & $\begin{array}{c}\text { Hyperdual numbers } \\
\text { (see in }[19,20,21,22,23,24])\end{array}$ & $\begin{array}{c}w=x_{1}+x_{2} \boldsymbol{\varepsilon}+y_{1} \varepsilon+y_{2} \boldsymbol{\varepsilon} \varepsilon \\
\boldsymbol{J}=\boldsymbol{\varepsilon}, \mathfrak{p}=0\end{array}$ \\
\hline & & & $\begin{array}{l}\text { Dual-hyperbolic numbers } \\
\text { (see in [18]) }\end{array}$ & $\begin{array}{c}w=x_{1}+x_{2} \mathbf{j}+y_{1} \varepsilon+y_{2} \mathbf{j} \varepsilon \\
J=\mathbf{j}, \mathfrak{p}=1\end{array}$ \\
\hline \multirow{3}{*}{$\mathbb{H} \mathbb{C}_{\mathfrak{p}}$} & \multirow{3}{*}{ 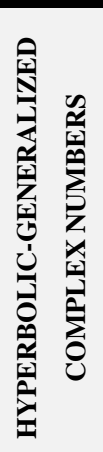 } & \multirow{3}{*}{$\begin{array}{c}3 \\
3 \\
+ \\
+ \\
+ \\
+ \\
+ \\
+ \\
+ \\
5 \\
11 \\
13\end{array}$} & $\begin{array}{c}\text { Hyperbolic-complex numbers } \\
(\text { see in }[8,9,10])\end{array}$ & $\begin{array}{c}\tilde{w}=x_{1}+x_{2} \mathbf{i}+y_{1} j+y_{2} \mathbf{i} j \\
J=\mathbf{i}, \mathfrak{p}=-1\end{array}$ \\
\hline & & & $\begin{array}{l}\text { Hyperbolic-dual numbers } \\
\text { (see in [18]) }\end{array}$ & $\begin{array}{c}\tilde{w}=x_{1}+x_{2} \boldsymbol{\varepsilon}+y_{1} j+y_{2} \boldsymbol{\varepsilon} j \\
J=\boldsymbol{\varepsilon}, \mathfrak{p}=0\end{array}$ \\
\hline & & & $\begin{array}{l}\text { Bihyperbolic numbers } \\
\text { (see in }[25,26,27,28])\end{array}$ & $\begin{array}{c}\tilde{w}=x_{1}+x_{2} \mathbf{j}+y_{1} j+y_{2} \mathbf{j} j \\
J=\mathbf{j}, \mathfrak{p}=1\end{array}$ \\
\hline \multirow{3}{*}{$\mathbb{C} \mathbb{C}_{\mathfrak{p}}$} & \multirow{3}{*}{ 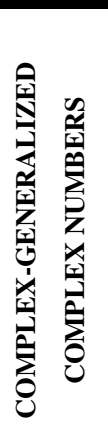 } & \multirow{3}{*}{ 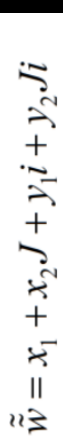 } & $\begin{array}{l}\text { Bicomplex numbers } \\
\text { (see in }[12,13,14])\end{array}$ & $\begin{array}{c}\tilde{\tilde{w}}=x_{1}+x_{2} \mathbf{i}+y_{1} i+y_{2} \mathbf{i} i \\
J=\mathbf{i}, \mathfrak{p}=-1\end{array}$ \\
\hline & & & $\begin{array}{l}\text { Complex-dual numbers } \\
\quad \text { (see in }[15,16,17]\end{array}$ & 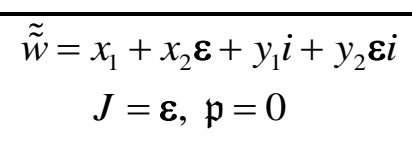 \\
\hline & & & $\begin{array}{c}\text { Complex-hyperbolic numbers } \\
(\text { see in }[8,9,10])\end{array}$ & $\begin{array}{c}\tilde{\tilde{w}}=x_{1}+x_{2} \mathbf{j}+y_{1} i+y_{2} \mathbf{j} i \\
J=\mathbf{j}, \mathfrak{p}=1\end{array}$ \\
\hline
\end{tabular}




\section{ACKNOWLEDGMENT}

The authors would like to thank the reviewers for their valuable comments and suggestions.

\section{CONFLICTS OF INTEREST}

No conflict of interest was declared by the authors.

\section{REFERENCES}

[1] Yaglom, I. M., Complex Numbers in Geometry, Academic Press, New York, (1968).

[2] Sobczyk, G., "The hyperbolic number plane", The College Mathematics Journal, 26(4): 268-280, (1995).

[3] Fjelstad, P., "Extending special relativity via the perplex numbers", American Journal of Physics, 54(5): 416-422 (1986).

[4] Study, E., Geometrie der Dynamen. Leipzig, (1903).

[5] Pennestrì, E., Stefanelli, R., "Linear algebra and numerical algorithms using dual numbers", Multibody System Dynamics, 18(3): 323-344 (2007).

[6] Majernik, V., "Multicomponent number systems", Acta Physica Polonica A, 90: 491-498, (1996).

[7] Harkin, A. A., Harkin, J. B., "Geometry of generalized complex numbers", Mathematics Magazine, 77(2): 118-129, (2004).

[8] Cockle, J., "On a new imaginary in algebra”, Philosophical magazine, London-Dublin-Edinburgh, 34(226): 37-47, (1849).

[9] Kantor, I., Solodovnikov, A., Hypercomplex Numbers, Springer-Verlag, New York, (1989).

[10] Alfsmann, D., "On families of $2^{\mathrm{N}}$-dimensional hypercomplex algebras suitable for digital signal processing", 14th European Signal Processing Conference (EUSIPCO 2006), Florence, Italy, (2006).

[11] Fjelstad, P., Sorin, G. Gal, “ $n$-dimensional hyperbolic complex numbers”, Advances in Applied Clifford Algebras, 8(1): 47-68, (1998).

[12] Price G. B., An introduction to multicomplex spaces and functions, New York: M. Dekker, (1991).

[13] Toyoshima H., "Computationally efficient bicomplex multipliers for digital signal processing", IEICE Transactions on Information and Systems, 81(2): 236-238, (1998).

[14] Rochon, D., Shapiro, M., "On algebraic properties of bicomplex and hyperbolic numbers", Analele Universitatii din Oradea. Fascicola Matematica, 11: 71-110, (2004).

[15] Cheng, H. H., Thompson, S., "Dual polynomials and complex dual numbers for analysis of spatial mechanisms", Proc. of ASME 24th Biennial Mechanisms Conference, Irvine, CA, August,19-22, (1996).

[16] Cheng, H. H., Thompson, S., "Singularity analysis of spatial mechanisms using dual polynomials and complex dual numbers", Journal of Mechanical Design, 121(2): 200-205, (1999).

[17] Messelmi, F., "Dual-complex numbers and their holomorphic functions", hal-01114178, (2015).

[18] Akar, M., Yüce, S., Şahin, S., "On the dual hyperbolic numbers and the complex hyperbolic numbers", Journal of Computer Science \& Computational Mathematics, 8 (1): 1-6, (2018). 
[19] Fike, J. A., "Numerically exact derivative calculations using hyper-dual numbers", 3rd Annual Student Joint Workshop in Simulation-Based Engineering and Design, (2009).

[20] Fike, J. A., Alonso J. J., "The development of hyper-dual numbers for exact second- derivative calculations", 49th AIAA Aerospace Sciences Meeting including the New Horizons Forum and Aerospace Exposition, Orlando, Florida, (2011).

[21] Fike, J. A., Jongsma, S., Alonso J. J., Weide E. Van Der, "Optimization with gradient and hessian information calculated using hyper-dual numbers", 29th AIAA Applied Aerodynamics Conference, Honolulu, Hawaii, (2011).

[22] Cohen, A., Shoham, M., "Principle of transference-An extension to hyper-dual numbers", Mechanism and Machine Theory, 125: 101-110, (2018).

[23] Fike, J. A., Alonso J. J., "Automatic differentiation through the use of hyper-dual numbers for second derivatives", Lecture Notes in Computational Science and Engineering book series (LNCSE), 87: 163-173, (2011).

[24] Fike, J. A., Alonso J. J., "Automatic differentiation through the use of hyper-dual numbers for second derivatives", 6th International Conference on Automatic Differentiation, Fort Collins, CO, July 23, (2012).

[25] Pogorui, A. A., Rodriguez-Dagnino, R. M., Rodrigue-Said, R .D., "On the set of zeros of bihyperbolic polynomials", Complex Variables and Elliptic Equations, 53(7): 685-690, (2008).

[26] Catoni, F., Boccaletti, D., Cannata, R., Catoni, V., Nichelatti, E., Zampetti, P., The mathematics of Minkowski space-time with an introduction to commutative hypercomplex numbers, Birkhauser Verlag, Basel, Boston, Berlin, (2008).

[27] Olariu, S., Complex Numbers in $n$-dimensions, North-Holland Mathematics Studies, Elsevier, Amsterdam, Boston, (2002).

[28] Bilgin, M., Ersoy, S., "Algebraic properties of bihyperbolic numbers", Advances in Applied Clifford Algebras, 30(1): 13, (2020).

[29] Apostolova, L. N., Krastev, K. I., Kiradjiev, B., "Hyperbolic double-complex numbers", AIP Conference Proceedings, 1184 (1): 193-198, (2009).

[30] Apostolova , L. N., Dimiev, S., Stoev, P., "Hyperbolic hypercomplex D-Bar operators, hyperbolic Cr-equations and harmonicity", arXiv:1012.3420v1, (2010).

[31] Özdemir, M., "Introduction to hybrid numbers", Advances in Applied Clifford Algebras, 28(1): 11, (2018). 